ISSN 1991-8631

Original Paper

http://indexmedicus.afro.who.int

\title{
Evaluation de neuf variétés de tomate (Solanum Lycopersicum L.) par rapport au flétrissement bactérien et à la productivité dans le Sud de la Côte d'Ivoire
}

\author{
Lassina FONDIO ${ }^{1 *}$, Hortense Andé DJIDJI ${ }^{1}$, François de Paul Mako N'GBESSO ${ }^{1}$ et \\ Daouda KONE ${ }^{2}$
}

${ }^{1}$ CNRA/Programme Cultures Maraîchères et Protéagineuses, 01BP633 Bouaké 01, Côte d'Ivoire.

${ }^{2}$ Université Félix Houphouët-Boigny, UFR Biosciences, 22BP582 Abidjan 22, Côte d'Ivoire.

*Auteur correspondant, E-mail: Ifondio@yahoo.fr

\section{RESUME}

Un essai d'évaluation de neuf variétés de tomate a été réalisé à la Station de recherche d'Anguédédou dans le Sud de la Côte d'Ivoire, en 2009, pour sélectionner des variétés résistantes au flétrissement bactérien et productives. L'essai a été disposé en bloc de Fisher avec 4 répétitions. Après une pépinière de 30 jours, les plants ont été repiqués en doubles lignes par planche de $5 \mathrm{~m}^{2}$ à raison de $0,5 \mathrm{~m}$ entre lignes et $0,5 \mathrm{~m}$ entre pied sur la ligne soit 20 pieds. Les observations et mesures ont porté sur la sensibilité des variétés au flétrissement bactérien, les délais de floraison, la durée de la production et les composantes du rendement. Les résultats indiquent que F1 Mongal (témoin) a été la plus productive. Les variétés CLN1466EA, CLN2418A, CLN2413L introduites de l'AVRDC et TMA97 du CNRA ont présenté des rendements bruts respectifs de 19,6, 22,2, 15,2 et 22,7 t/ha avec une faible sensibilité au flétrissement bactérien, mais un fort taux d'avarie (19 à 53,4\%) de fruits. Pour améliorer la performance de ces variétés, il paraît indiqué de poursuivre les recherches dans une approche agronomique visant la maîtrise de l'eau d'irrigation, une gestion intégrée des ravageurs et maladies pendant le cycle cultural.

(C) 2013 International Formulae Group. All rights reserved.

Mots clés : Rendement, avarie, performance, humidité, résistance, maladies.

\section{INTRODUCTION}

La tomate constitue, après la pomme de terre, le deuxième légume frais ou transformé, le plus consommé dans le monde (INRA, 2010). Selon cette source, la production mondiale de tomate a connu une forte croissance depuis 1978 où elle est passée de 48 millions de tonnes à 74 millions en 1992 , et de 89 millions de tonnes en 1998 pour atteindre 124 millions en 2006. Selon la FAO (2013), la Chine, les Etats-Unis et la Turquie en sont les plus grands pays producteurs, avec $48,58,12,6$ et 11 millions de tonnes de tomate fraîche par an, contre 32364 tonnes pour la Côte d'Ivoire. Cette production demeure faible pour répondre aux besoins des populations ivoiriennes estimés à plus de 100000 tonnes de tomate par an (Soro et al., 2007).

De nombreux facteurs peuvent être évoqués pour expliquer la faiblesse de la production de cette culture en Côte d'Ivoire. Il s'agit principalement de la forte sensibilité aux facteurs biotiques de certaines variétés commercialisées sur le marché et du climat chaud et humide qui favorise le 
développement des maladies et ravageurs. Quant aux variétés les plus adaptées au climat, elles ne répondent pas toujours aux besoins des producteurs et consommateurs (faible productivité, goût aigre, etc.). Au niveau des facteurs biotiques, les maladies virales, fongiques et bactériennes constituent les principaux fléaux au développement de la tomate dans ce pays. Concernant les maladies bactériennes, le flétrissement bactérien dû à Ralstonia solanacearum est devenu une préoccupation majeure pour les producteurs surtout ceux des grandes villes comme Abidjan où le maraîchage est confronté à des problèmes de terres cultivables. A cet effet, des producteurs de la Sous-Préfecture de Songon près d'Abidjan ont affirmé avoir abandonné la culture de la tomate à cause du flétrissement bactérien qui peut leur causer 80 à $100 \%$ de perte de rendement selon les variétés de tomate (Fondio et al., 2010). Pour lutter contre cette maladie bactérienne, des techniques agronomiques préconisant l'observation de jachère ou la rotation culturale de la tomate avec d'autres cultures n'appartenant pas à la famille des Solanacées, semblent montrer, aujourd'hui, des limites dans les zones urbaines et périurbaines à cause $\mathrm{du}$ fort déficit foncier. Les producteurs sont obligés d'exploiter de façon continue la même portion de terre sans observer de jachère. En conséquence, la recherche de variétés tolérantes à cette maladie et productives paraît la solution indiquée pour aider les producteurs à améliorer la productivité de leur culture de tomate. C'est dans ce contexte que cette étude est proposée en vue d'identifier des variétés productives de tomate tolérantes au flétrissement bactérien.

\section{MATERIEL ET METHODES \\ Site d'étude}

L'essai a été conduit à la Station d'Expérimentation et de Production du Centre National de Recherche Agronomique (CNRA) d'Anguédédou $\left(5^{\circ} 22\right.$ de Latitude Nord, $4^{\circ} 8$ de Longitude et $95 \mathrm{~m}$ d'Altitude) située à $30 \mathrm{~km}$ à l'Ouest d'Abidjan sur l'axe routier AbidjanDabou.

Le climat de la zone d'étude est de type bimodal avec deux saisons de pluies (marsjuin et septembre-novembre) et deux saisons sèches (juillet-août et décembre-février). Au cours de la période de l'essai, la température moyenne mensuelle a varié entre 27 et $31{ }^{\circ} \mathrm{C}$ et l'hygrométrie moyenne mensuelle a été de $73,6 \%$. La Figure 1 présente les histogrammes de la pluviométrie et les courbes des températures maxi et mini de l'année 2009 à Anguédédou. L'essai s'est déroulé au cours de la petite saison des pluies, de septembre à novembre 2009, qui a enregistré $317,7 \mathrm{~mm}$. Ce climat chaud et humide qui a ainsi prévalu au cours de l'essai. Le sol de la parcelle expérimentale est ferralitique et à texture sableuse. Une analyse d'échantillons de ce sol, réalisée par le Laboratoire de Pédologie de l'Ecole Supérieure d'Agronomie de Yamoussoukro, a révélé qu'il est fortement acide avec une matière organique bien décomposée (Tableau 1).

\section{Matériel végétal}

Le matériel végétal était composé de 9 variétés de tomate dont cinq introduites de l'Asian Vegetable Research and Development Center (AVRDC)-The World Vegetables Center, en Taïwan, une variété de l'Institut National de Recherche Agronomique (INRA) de France, deux variétés commerciales Caraïbo et l'hybride F1 Mongal, et la variété TMA97 sélectionnée au CNRA de Côte d'Ivoire (Tableau 2). La variété F1 Mongal qui sert de témoin est largement vulgarisée auprès des producteurs par les firmes semencières en Côte d'Ivoire.

\section{Méthodes}

\section{Pratiques culturales}

La pépinière a été réalisée en Août 2009 sur une planche de $3 \mathrm{~m}$ de long et $1 \mathrm{~m}$ de large. La planche a été désinfectée avec du carbofuran (Furadan) à raison de $50 \mathrm{~g} / \mathrm{m}^{2}$. Ensuite, il a été apporté $20 \mathrm{~g} / \mathrm{m}^{2}$ de NPK 10 18-18. Les graines de tomate ont été semées 
en lignes distantes de $10 \mathrm{~cm}$ avec $5 \mathrm{~cm}$ entre les poquets sur la ligne. Après le semis, la planche a été recouverte avec des feuilles de palmier à huile. Celles-ci ont été d'abord déposées à même le sol pendant les 3 premiers jours après le semis. Ensuite, elles ont été relevées pour monter une ombrière à une hauteur de $80 \mathrm{~cm}$ du sol. A une semaine du repiquage, l'ombrière a été allégée, en réduisant la quantité de palme. A deux jours du repiquage, l'ombrière a été complètement enlevée. La pépinière était régulièrement arrosée. Pour le repiquage, le sol a été labouré manuellement et $300 \mathrm{~kg} / \mathrm{ha}$ d'engrais minéral NPK 10-18-18 ont été apportés comme fumure de fond. Dans les poquets de repiquage, $20 \mathrm{~g}$ de carbofuran ont été de nouveau apportés pour désinfecter le sol contre les champignons. Les plants ont été repiqués en septembre 2009 après un mois de pépinière. Pendant la culture, en fumure d'entretien aux deuxièmes et cinquièmes semaines après le repiquage, un mélange de $100 \mathrm{~kg} / \mathrm{ha}$ d'urée et $200 \mathrm{~kg} / \mathrm{ha}$ de Sulfate de potasse a été apporté. Des traitements phytosanitaires au Deltaméthrine (11/ha) et au Manèbe (3,5 kg/ha) ont été effectués tous les 15 jours. Pendant la culture, six sarclages ont été réalisés pour éliminer les mauvaises herbes.

\section{Dispositif expérimental}

L'essai a été disposé en bloc de Fisher avec 4 répétitions. La parcelle élémentaire était constituée d'une planche de $5 \mathrm{~m}$ de long et de $1 \mathrm{~m}$ de large. Les plants ont été repiqués sur la planche en doubles lignes à raison de 0,5 m entre pieds et $0,5 \mathrm{~m}$ entre lignes soit 20 plants par parcelle élémentaire.

\section{Observations et mesures}

Les observations et mesures ont porté sur : la sensibilité au flétrissement bactérien, la taille des plants au stade de floraison, les dates des stades phénologiques et les composantes du rendement.

La sensibilité au flétrissement bactérien a été déterminée par comptage hebdomadaire des plants flétris. Ce comptage a commencé une semaine après le repiquage et a pris fin au stade de la fructification. Pour s'assurer que les plants de tomate sont effectivement morts du flétrissement bactérien (Figure 2), nous avons utilisé le test du kit LFD (Lateral Flow Device) qui est une solution spécifique à Ralstonia solanacearum, la bactérie responsable du flétrissement des plants. Ce test consiste à déposer une goutte de solution à base d'extrait d'un broyat d'une partie de la tige du plant flétri sur du papier buvard contenant la solution spécifique du Ralstonia. La présence de la bactérie est détectée par un virement au bleu. En cas d'absence de la bactérie dans la tige, il n'y a aucun virement de couleur.

La taille des plants a été déterminée par la mesure de tous les plants au stade de la floraison. Les dates des stades phénologiques notées ont porté sur le délai de floraison $(50 \%$ des plants de la parcelle ayant fleuri), la date de première récolte et la date de dernière récolte des plants par parcelle élémentaire. Les composantes du rendement déterminées ont été le nombre de fruits récoltés, le nombre de fruits sains et de fruits avariés, le poids total des fruits, le poids des fruits sains et le poids des fruits avariés. Pour déterminer ces composantes du rendement, l'addition $\mathrm{du}$ nombre et du poids total des fruits de toutes les récoltes a été effectuée. Le nombre et le poids des fruits sains ont été déterminés par la déduction du nombre et du poids total des fruits récoltés, le nombre et le poids des fruits avariés non commercialisables. Avec le poids total et le poids net, les rendements bruts et nets ont été calculés. La variable fruits avariés comprend les fruits perforés, éclatés, pourris, etc.

\section{Analyse des données \\ Les résultats des observations et mesures ont été analysés au Logiciel SAS System for Windows Version 9.0 (SAS, 2002). Une analyse de variance a été d'abord effectuée. Ensuite, les moyennes ont été séparées par la méthode de Duncan au seuil de $5 \%$ quand les effets étaient significatifs.}


Tableau 1 : Composition chimique du sol de la parcelle expérimentale d'évaluation des variétés de tomate.

\begin{tabular}{ccccccccc}
\hline pH & $\mathbf{C} / \mathbf{N}$ & \multicolumn{2}{c}{ Phosphore (ppm) } & \multicolumn{5}{c}{ Complexe absorbant $(\mathbf{c m o l} / \mathbf{k g})$} \\
\cline { 2 - 8 } & & $\mathbf{P}$ total & $\mathbf{P}$ ass. & $\mathbf{C E C}$ & $\mathbf{C a}^{2+}$ & $\mathbf{M g}^{2+}$ & $\mathbf{K}^{+}$ & $\mathbf{N a}^{+}$ \\
\hline 4,5 & 10,5 & 313 & 100 & 4,72 & 0,322 & 0,129 & 0,072 & 0,039 \\
\hline
\end{tabular}

Tableau 2 : Liste des variétés de tomate évaluées à la Station d'Anguédédou en 2009.

\begin{tabular}{lcc}
\hline $\mathbf{N}^{\circ}$ & Noms des accessions & Origines des accessions \\
\hline 1 & CLN2418A & AVRDC \\
2 & CLN1466EA & AVRDC \\
3 & CLN1460A & AVRDC \\
4 & CLN2413L & AVRDC \\
5 & CLN1464A & AVRDC \\
6 & Caraïbo & INRA \\
7 & LignonC8-6M2-71 & INRA \\
8 & F1 Mongal & Commerciale \\
9 & TMA97 & CNRA \\
\hline
\end{tabular}

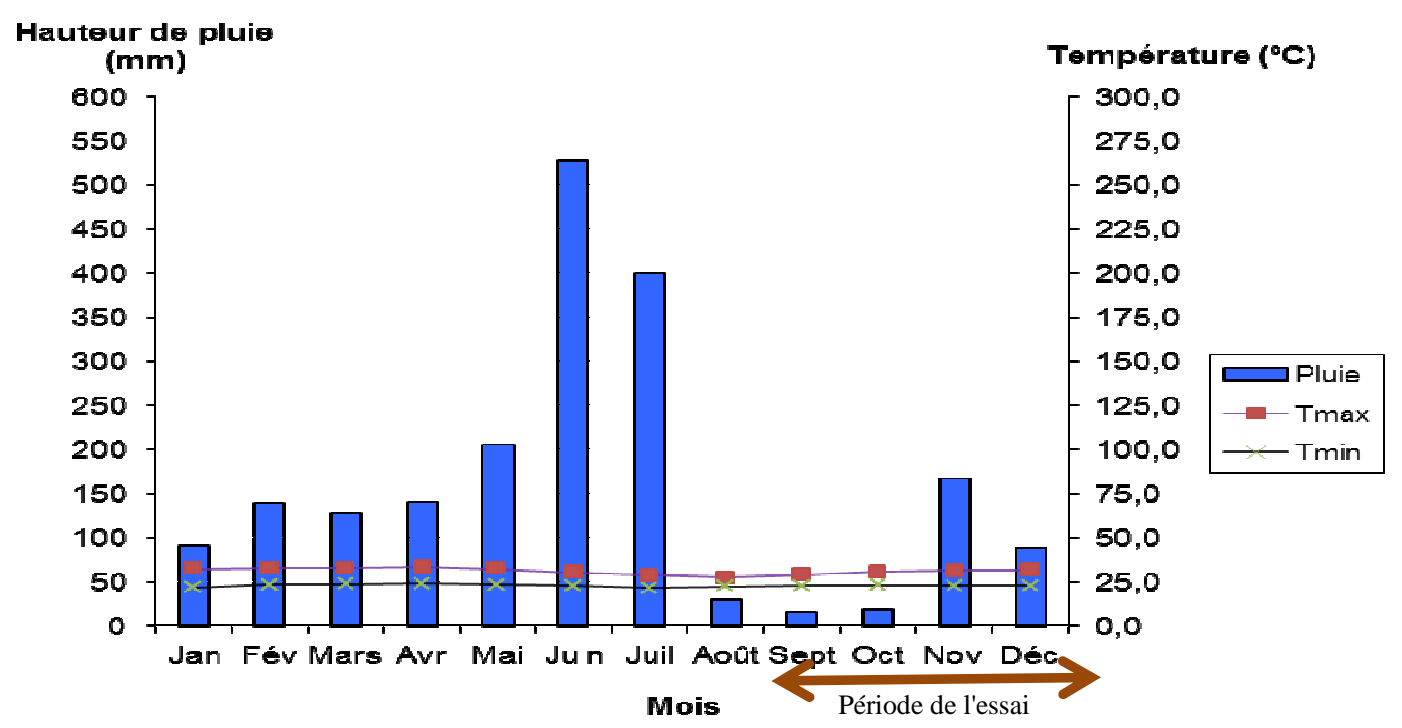

Figure 1: Pluviométrie mensuelle, températures maxi et mini enregistrées à la Station d'Anguédédou en 2009. 


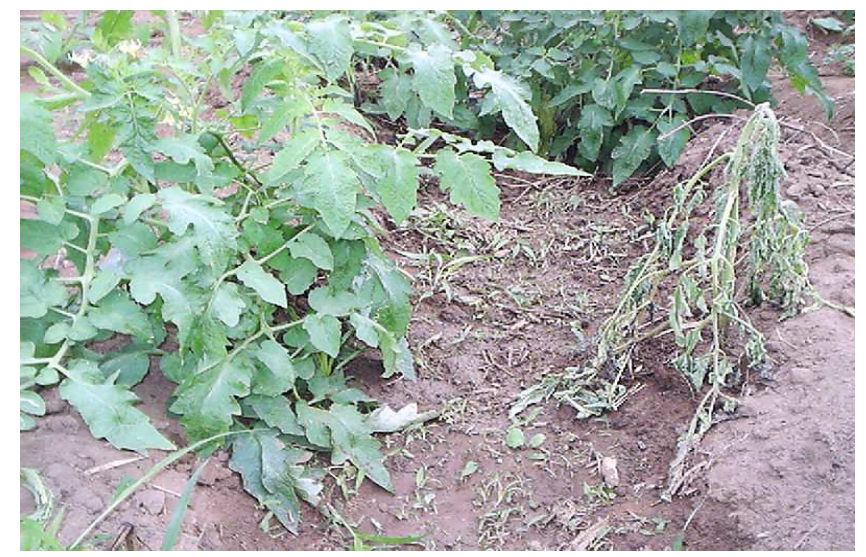

Figure 2 : Un plant de tomate de la variété TMA97 mort du flétrissement bactérien.

\section{RESULTATS}

Sensibilité des plantes de tomate au flétrissement bactérien

Selon le Tableau 3, une différence significative entre les variétés de tomate par rapport à la sensibilité au flétrissement bactérien est notée. Les taux de plants flétris ont varié entre $28,6 \%$ et $57,2 \%$. Les lignés TMA97, CLN2413L, LignonC8-6M2-71 et F1 Mongal (témoin) ont été les moins sensibles avec un taux de mortalité de $28,6 \%$. Les lignés CLN1466EA, CLN2418A, Caraïbo et CLN1464A ont été classées comme moyennement sensibles au flétrissement bactérien avec un taux de mortalité oscillant entre 33,3 et $42,9 \%$. Avec $57,2 \%$ de mortalité, la variété CLN1460A a été la plus sensible.

Taille, délai de floraison, délai de première récolte et durée de la production des plants de tomate

La différence entre les variétés de tomate est significative pour la taille des plants, le délai de floraison, le délai de première récolte et la durée de la production (Tableau 4). La taille des plants au stade de la floraison a varié entre 32 et $51 \mathrm{~cm}$. La plus petite taille des plants $(32 \mathrm{~cm})$ a été obtenue avec la variété CLN2418A et la plus grande taille $(51 \mathrm{~cm})$ avec CLN1466EA. Les plantes ont fleuri entre 58 jours après semis (Jas) avec la variété LignonC8-6M2-71 et $45 \mathrm{Jas}$ avec la variété commerciale Caraïbo. Par contre, le délai de première récolte a évolué entre 94 Jas avec les variétés CLN2418A et LignonC86M2-71 et 111 Jas avec CLN1460A. La durée de la production a été 9 à 25 jours avec les variétés Caraïbo et CLN2418A.

\section{Taux d'avarie des fruits et rendements des variétés de tomate}

La différence entre les variétés de tomate est significative pour le taux d'avarie, le rendement brut et le rendement net en fruits (Tableau 5). Le taux d'avarie a varié entre $11,9 \%$ avec la variété F1 Mongal et 99,3\% avec la variété LignonC8-6M2-71. La variété TMA97 sélectionnée au CNRA a enregistré un taux d'avarie de $53,4 \%$. Concernant le rendement brut, ce caractère a oscillé entre 8,5 t/ha (Caraïbo) et $48 \mathrm{t} / \mathrm{ha}$ (F1 Mongal). Le rendement net a, par contre, évolué entre 0,2 t/ha avec la variété LignonC8-6M2-71 et 42,7 t/ha avec F1 Mongal qui a enregistré le rendement le plus élevé. Les variétés les plus productives obtenues de l'AVRDC ont été CLN2418A et CLN1466EA qui ont obtenu 16,2 et 16,5 t/ha de rendement net. La variété TMA97 du CNRA a obtenu un rendement net de $10,7 \mathrm{t} / \mathrm{ha}$. 
Tableau 3 : Taux de plants par variétés de tomate évaluées à la Station d'Anguédédou en 2009.

\begin{tabular}{lc}
\hline Variétés de tomate & $\begin{array}{c}\text { Taux de pieds flétris au stade de la fructification } \\
(\boldsymbol{\%})\end{array}$ \\
\hline CLN1466EA & $33,3 \mathrm{ab}$ \\
Caraïbo & $38,1 \mathrm{ab}$ \\
TMA97 & $28,6 \mathrm{c}$ \\
F1 Mongal & $28,6 \mathrm{c}$ \\
CLN1464A & $42,9 \mathrm{~b}$ \\
CLN1460A & $57,2 \mathrm{a}$ \\
CLN2413L & $28,6 \mathrm{c}$ \\
CLN2418A & 33,3 ab \\
LignonC8-6M2-71 & $28,6 \mathrm{c}$ \\
Moyenne & 34,8 \\
P (\%) & 0,0005 \\
CV (\%) & 9,6 \\
\hline$*$ Les nombres en colonnes, affectés de la même lettre, ne diffèrent pas significativement au seuil de 5\% \\
(Test de Duncan).
\end{tabular}

Tableau 4: Taille des plants à la floraison, délai de floraison, délai de première récolte et durée de la production des variétés de tomate évaluées à la Station d'Anguédédou en 2009.

\begin{tabular}{lcccc}
\hline Variétés de tomate & $\begin{array}{c}\text { Taille des } \\
\text { plants stade } \\
\text { floraison }(\mathbf{c m})\end{array}$ & $\begin{array}{c}\text { Délai de } \\
\text { floraison } \\
\left(\mathbf{J a s}^{\mathbf{1}}\right)\end{array}$ & $\begin{array}{c}\text { Délai de } \\
\text { première récolte } \\
(\mathbf{J a s})\end{array}$ & $\begin{array}{c}\text { Durée de la } \\
\text { production }(\mathbf{j})\end{array}$ \\
\hline CLN1466EA & $51 \mathrm{a}^{*}$ & $62 \mathrm{bcd}^{*}$ & $102 \mathrm{bcd}$ & $13 \mathrm{~b}$ \\
Caraïbo & $45 \mathrm{ab}$ & $71 \mathrm{a}$ & $107 \mathrm{ab}$ & $9 \mathrm{~b}$ \\
TMA97 & $41 \mathrm{~b}$ & $64 \mathrm{bcd}$ & $99 \mathrm{bcd}$ & $13 \mathrm{~b}$ \\
F1 Mongal & $41 \mathrm{~b}$ & $59 \mathrm{~cd}$ & $97 \mathrm{~cd}$ & $18 \mathrm{ab}$ \\
CLN1464A & $41 \mathrm{~b}$ & $64 \mathrm{abc}$ & $105 \mathrm{abc}$ & $11 \mathrm{~b}$ \\
CLN1460A & $41 \mathrm{~b}$ & $66 \mathrm{abc}$ & $111 \mathrm{a}$ & $9 \mathrm{~b}$ \\
CLN2413L & $40 \mathrm{~b}$ & $68 \mathrm{ab}$ & $102 \mathrm{bcd}$ & $15 \mathrm{~b}$ \\
CLN2418A & $32 \mathrm{c}$ & $59 \mathrm{~cd}$ & $94 \mathrm{~d}$ & $25 \mathrm{a}$ \\
LignonC8-6M2-71 & $37 \mathrm{~b}$ & $58 \mathrm{~d}$ & $94 \mathrm{~d}$ & $19 \mathrm{ab}$ \\
Moyenne & 41 & 63,4 & 101 & 14,6 \\
P $(\%)$ & 0,014 & 0,006 & 0,004 & 0,02 \\
CV $(\%)$ & 12,6 & 9,4 & 4,6 & 36,6 \\
\hline
\end{tabular}

* Les nombres en colonnes, affectées de la même lettre, ne diffèrent pas significativement au seuil de 5\% (Test de Duncan). ${ }^{1}$ Jas : Jours après semis. 
Tableau 5 : Taux d'avarie des fruits et rendements des variétés de tomate évaluées à la Station d'Anguédédou en 2009.

\begin{tabular}{lccc}
\hline Variétés de tomate & $\begin{array}{c}\text { Taux d'avarie des fruits } \\
(\boldsymbol{\%})\end{array}$ & $\begin{array}{c}\text { Rendement brut } \\
(\mathbf{t} / \mathbf{h a})\end{array}$ & $\begin{array}{c}\text { Rendement net } \\
(\mathbf{t} / \mathbf{h a})\end{array}$ \\
\hline CLN1466EA & $19,0 \mathrm{e}^{*}$ & $19,6 \mathrm{~b}$ & $16,5 \mathrm{~b}$ \\
Caraïbo & $16,6 \mathrm{e}$ & $8,5 \mathrm{c}$ & $7,7 \mathrm{~d}$ \\
TMA97 & $53,4 \mathrm{bc}$ & $22,7 \mathrm{~b}$ & $10,7 \mathrm{bc}$ \\
F1 Mongal & $11,9 \mathrm{e}$ & $48,0 \mathrm{a}$ & $42,7 \mathrm{a}$ \\
CLN1464A & $60,3 \mathrm{~b}$ & $19,4 \mathrm{~b}$ & $8,6 \mathrm{bc}$ \\
CLN1460A & $32,3 \mathrm{~d}$ & $8,7 \mathrm{c}$ & $7,3 \mathrm{~cd}$ \\
CLN2413L & $46 \mathrm{c}$ & $15,2 \mathrm{bc}$ & $10,5 \mathrm{bc}$ \\
CLN2418A & $28,2 \mathrm{de}$ & $22,2 \mathrm{~b}$ & $16,2 \mathrm{~b}$ \\
LignonC8-6M2-71 & $99,3 \mathrm{a}$ & $18,4 \mathrm{bc}$ & $0,2 \mathrm{~d}$ \\
Moyenne & 40,7 & 20,3 & 13,4 \\
P $(\%)$ & 0,001 & 0,0001 & 0,001 \\
CV $(\%)$ & 35,5 & 26,7 & 32,4 \\
\hline * Lesnombres en colonnes, affectées de la même lettre, ne diffèrent pas significativement au seuil de 5\% (Test de Dun
\end{tabular}

\section{DISCUSSION}

\section{Sensibilité au flétrissement bactérien}

La différence significative observée entre les variétés de tomate par rapport au flétrissement bactérien indique que la sensibilité à cette maladie dépend des variétés donc du matériel végétal. N'guessan et al. (2012) ont montré qu'il existe différents niveaux de sensibilité au flétrissement bactérien chez la tomate allant des variétés hautement sensibles au plus résistantes en Côte d'Ivoire. Par exemple, F1 Mongal (le témoin) est connue pour sa résistance au flétrissement bactérien et son adaptation au climat chaud et humide (Van Der Vossen et al., 2004). Les variétés introduites de l'AVRDC ayant enregistré le même taux de flétrissement que F1 Mongal peuvent donc être considérées comme tolérantes à cette maladie. Par contre, celles ayant obtenu le plus grand taux de plants flétris sont ainsi les plus sensibles. Cependant, l'étendue de la maladie du flétrissement bactérien à toutes les variétés de la parcelle expérimentale pourrait s'expliquer par les conditions climatiques chaudes et humides qui ont favorisé la multiplication et la dissémination de la bactérie. En effet, les valeurs de 27 à $31{ }^{\circ} \mathrm{C}$ de température moyenne et $73,6 \%$ d'hygrométrie moyenne observées lors de l'étude, sont favorables au développement de la bactérie responsable du flétrissement de la tomate. Selon Cariglia (2007), les températures audessus de $25^{\circ} \mathrm{C}$ et l'excès d'eau favorisent la multiplication et la progression de la maladie. En outre, cet auteur a noté que les sols sableux, limoneux et argileux facilitent le développement de Ralstonia solanacearum. Il serait vraisemblable de dire que la texture sableuse du sol de la parcelle expérimentale a contribué à la prolifération de la bactérie dont les germes ont été ensuite disséminés à toutes les plantes de la parcelle par le ruissellement des eaux de pluies. La bactérie peut être disséminée facilement par les eaux de surface lorsque des racines affectées par la maladie se développent dans de l'eau stagnante (Janse, 1996 ; Poussier, 2000; Van Elsas et al., 2001). Ce résultat confirme donc le rôle d'un bon drainage des parcelles comme moyen de gestion du flétrissement bactérien en culture de la tomate (Naika et al., 2005). 
Délai de floraison, de première récolte, durée de la production et composantes du rendement

La différence significative observée entre les variétés de tomate pour les délais de floraison et de première récolte est liée à la différence entre leur cycle de culture. Les variétés à cycle court fleurissent précocement et celles à cycle long tardivement. Cependant, cette observation ne semble pas être confirmée pour toutes les cultures. En effet, Nieuwenhuis et Nieuwelink (2005) ont observé chez le soja que des variétés très précoces fleurissent à 30-35 jours après le semis et murissent à 75 à 105 jours. et les variétés tardives fleurissent également à 30-35 jours après semis mais murissent à 110 à 140 jours. Par contre, la différence entre les variétés pour la durée de la production peut être due à des facteurs extérieurs dont les attaques de maladies fongiques, bactériennes ou virales qui peuvent agir sur le développement des plantes réduisant ainsi leur capacité de production. Les rendements bruts de 15,2 à 22,7 t/ha obtenus par CLN1466EA, CLN2418A, CLN2413L et TMA97 indiquent que ces variétés possèdent une grande capacité de production. Le fort taux d'avarie des fruits (19 à 53,4\%) subi par la majorité des fruits serait lié, d'une part, aux fortes pluies enregistrées au cours de l'essai en novembre 2009 (Figure 1) qui ont favorisé le phénomène de pourriture apicale après l'éclatement de l'apex des fruits, et d'autre part, dans une moindre mesure, aux dégâts des ravageurs et maladies. En effet, selon Lacroix (1999), la pourriture apicale des fruits de tomate peut être provoquée par une absorption excessive de calcium dans les cellules suite à un excès d'eau d'irrigation. Le taux de 53,4\% d'avarie enregistré par la variété TMA97 du CNRA dans cette étude confirme la sensibilité de cette variété à la pourriture apicale des fruits observée dans des essais antérieurs à la Station d'Anguédédou (Fondio et al., 2010). Ces avaries qui entrainent des rejets de fruits peuvent expliquer les faibles niveaux de rendements nets (10,5 à 16,5 t/ha) affichés par les variétés CLN1466EA, CLN2418A, CLN2413L et TMA97. Pour améliorer le rendement net de ces variétés, il paraît bien indiqué d'agir sur les facteurs responsables des avaries de fruits notamment le phénomène de pourriture apicale suite à l'éclatement des fruits, perforations des fruits, etc. Pour ce faire, les recherches sur ces variétés pourraient être poursuivies dans une approche agronomique visant la maîtrise de l'eau d'irrigation, une gestion intégrée des ravageurs et maladies pendant le cycle cultural.

\section{Conclusion}

Il ressort de cette étude que 4 variétés de tomate, TMA97, CLN2413L, Lignon C86M2-71 et F1 Mongal, se sont révélées tolérantes au flétrissement avec 28,6\% de mortalité des plants. Pour le rendement, la variété commerciale F1 Mongal a été la plus productive grâce à son adaptation au climat et sa résistance aux facteurs biotiques. Les variétés CLN1466EA, CLN2418A, CLN2413L introduites de l'AVRDC et TMA97 du CNRA qui ont présenté les meilleurs rendements bruts 15,2 à 22,7 t/ha avec une faible sensibilité au flétrissement bactérien, sont caractérisées par de forts taux d'avarie (19 à 53,4\%) essentiellement dus à la pourriture apicale.

En perspective, il faudra poursuivre les recherches sur les variétés CLN1466EA, CLN2418A, CLN2413L et TMA97 afin de lever la contrainte liée aux forts taux d'avarie des fruits. La mise au point de techniques de la maîtrise de l'eau d'irrigation pendant la phase de fructification des plants et de lutte contre les maladies et ravageurs pourraient être explorées comme solutions.

\section{REFERENCES}

Cariglia A. 2007. Lutte préventive contre le flétrissement bactérien en culture de la tomate hors-sol - Etat des connaissances et conseils. Pôle de protection des plantes, Réunion, St-Pierre. 
FAO. 2013. Production Mondiale de la Tomate en 2011. FAOSTAT, FAO: Rome, Italie.

Fondio L, Djidji AH, N'gbesso MFDP, Ochou OG. 2010. Evaluation des variétés de tomate et piment pour la tolérance au flétrissement bactérien, et multiplication des semences de piment. Rapport d'activités, Convention CNRA/AVRDC, CNRA, Bouaké, Côte d'Ivoire, 31p.

INRA. 2010. Légumes. Ephytia.inra.fr/ tomate/importance économique.

Jane JD. 1996. Potato brown rot in Western Europe - History, present occurrence and some remarks on possible origin, epidemiology and control strategies. Bull. OEPP/EPP Bull., 26: 679-695.

Lacroix M. 1999. Nutrition en calcium : problèmes et prévention. Ministère de l'Agriculture, des Pêches et de l'Alimentation, Publications VT 046, Quebec, Canada, 15p.

N'guessan CA, Abo K, Fondio L, Chiroleu F, Lebeau A, Poussier S, Wicker E, Koné D. 2012. So near and yet so far: the specific case of Ralstonia solanacearum populations from Côte d'Ivoire in Africa. Phytopathology, 102: 733-740.

Naika S, Van Dam B, Florijn A. 2005. La Culture de la Tomate, Production, Transformation et Commercialisation $\left(5^{e}\right.$ édn révisée). Agromisa Foundation, coll. Agrodok: Wageningen ; 17.
Nieuwenhuis R, Nieuwenlink J. 2005. La Culture du Soja et d'autres Légumineuses ( $2^{\text {ème }} e d n$ ). Agromisa Foundation, coll. Agrodok 10 : Wageningen ; 74 p.

Poussier S. 2000. Exploration de la diversité génétique de Ralstonia solanacearum, agent du flétrissement bactérien. Détection et dynamique dans les réservoirs d'inoculum. Ph.D thesis, Rennes I, Rennes, France, 149 p.

SAS. 2002. SAS System for Window V9.0. SAS Institute Inc., NC: USA.

Soro S, Doumbia M, Dao D, Tschannen A, Girardin O. 2007. Performance de six cultivars de tomate Lycopersicon esculentum Mill. Contre la jaunisse en cuillère des feuilles, le flétrissement bactérien et les nématodes à galles. Sciences \& Nature, 4(2): 123-130.

Van der Vossen HAM, Nono-Womdim R, Messiaen CM. 2004. Lycopersicon esculentum Mill. In PROTA 2: Vegetables/Légumes, Grubben, GJH, Denton OA (eds). PROTA: Wageningen, Pays Bas.

Van Elsas JD, Kastelein P, De Vries PM, Van Overbeek LS. 2001. Effects of ecological factors on the survival of Rasltonia solanacearum bv.2 in irrigation water. Revue Canadienne de Microbiologie, 47(9): 842-854. 